\title{
Review
}

\section{Transcriptional regulation of lung development: emergence of specificity Parviz Minoo}

\author{
University of Southern California School of Medicine, Los Angeles, California, USA
}

Received: 4 August 2000

Revisions requested: 14 August 2000

Revisions received: 24 August 2000

Accepted: 24 August 2000

Published: 1 September 2000
Respir Res 2000, 1:109-115

(c) Current Science Ltd (Print ISSN 1465-9921; Online ISSN 1465-993X)

\begin{abstract}
The lung is the product of a set of complex developmental interactions between two distinct tissues, the endodermally derived epithelium and the mesoderm. Each tissue contributes to lung development by fine-tuning the spatial and temporal pattern of gene expression for a distinct array of signaling molecules, transcriptional molecules and molecules related to the extracellular matrix. Morphoregulatory transcriptional factors such as NKX2.1 have the crucial role of connecting the cell-cell crosstalk to the activation or repression of gene expression through which processes such as cellular proliferation, migration, differentiation and apoptosis can be controlled. Although none of the factors participating in lung development are exclusively lung-specific, their unique combinations and interactions constitute the basis for emergence of lung structural and functional specificities. An understanding of the individual molecules and their unique interactions in the context of lung development is necessary for the construction of a morphogenetic map for this vital organ as well as for the development of rational and innovative approaches to congenital and induced lung disease.
\end{abstract}

Keywords: alveolar type I cell, alveolar type II cell, Bmp4, epithelial-mesenchymal interactions, extracellular matrix protein, Fgf-10, growth factors, Hnf3, lung morphogenesis, morphogens, Nkx2.1, Shh, transcription factors

\section{Introduction}

Temporal and spatial control of gene expression by transcriptional factors is a hallmark of development. The lung is no exception to this, and the program of lung development from its inception is directed by the activity of key transcriptional factors. The present review is a process-based rather than a gene-based perspective on the role of morphoregulatory transcription factors in lung development. Reference is also made to the morphogenetic signaling molecules that might be involved in modulating transcrip- tional factors. Although lung development is a continuum, for simplicity it can be viewed as occurring in four phases: the first is the specification of the lung primordium; next comes the formation of the trachea and septation from the esophagus; after this is branching morphogenesis and establishment of the infrastructure for the differentiation of specialized epithelial cells along the proximo-distal (P-D) axis; and finally alveologenesis and differentiation of distal epithelial cell types, alveolar type I and type II cells, take place (Fig. 1).

BMP-4 = bone morphogenetic protein-4; ECM = extracellular matrix; FGF = fibroblast growth factor; Gli = glial nuclear protein; HFH = hepatocyte nuclear factor-3/forkhead homolog; HNF-3 = hepatocyte nuclear factor-3; P-D = proximo-distal; PDGF = platelet-derived growth factor; RAR = retinoic acid receptor; $\mathrm{SHH}=$ Sonic hedgehog. 
Figure 1

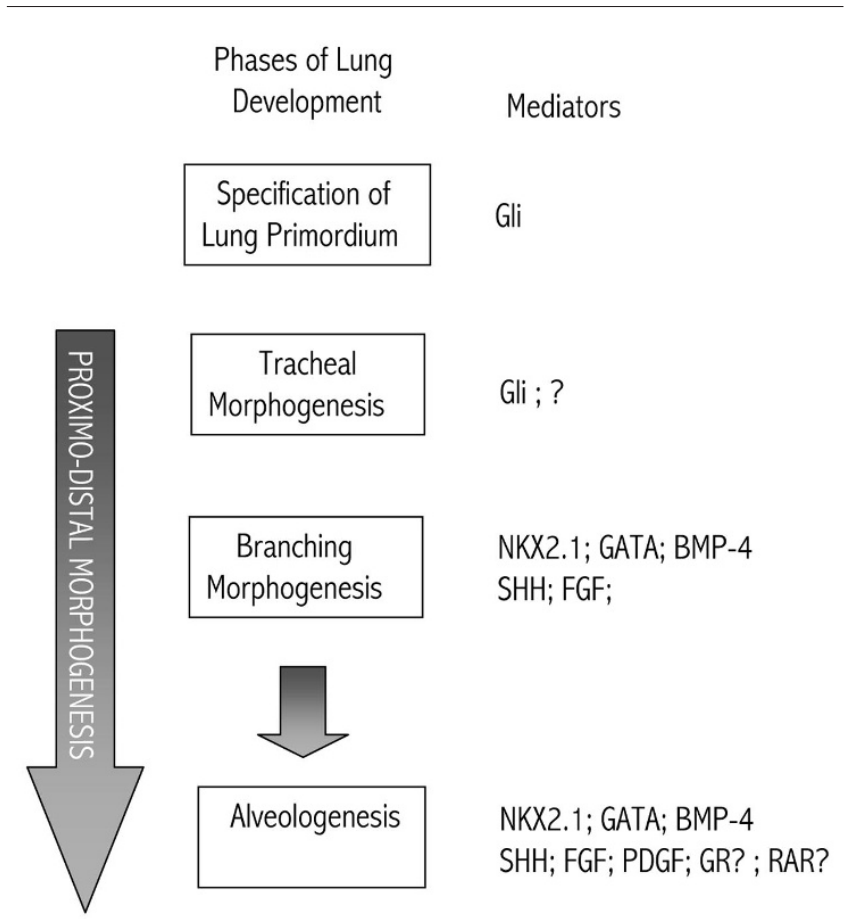

Four arbitrary phases in lung development and the key mediators. GR, glucocorticoid receptor.

In general, morphoregulatory transcriptional factors constitute an operational linkage between signaling and cellular behavior, as illustrated by the simple paradigm signaling $\rightarrow$ transcription factors $\rightarrow \rightarrow \rightarrow$ cellular behavior.

In the lung, signaling is mediated by peptide growth factors such as fibroblast growth factor (FGF) and bone morphogenetic protein-4 (BMP-4), as well as by the morphogens Sonic hedgehog $(\mathrm{SHH})$ and retinoids. Morphogenetic signaling originating from the cell's environment is translated into cellular behavior through changes in the activity of transcription factors such as hepatocyte nuclear factor-3 (HNF-3), NKX2.1 and GATA6. Among these, the role of NKX2.1 as a transcriptional regulator of lung development is somewhat better understood, permitting a more detailed discussion. By definition, transcription factors modulate the activity of downstream target genes, so the potential targets are presumably those encoding members of the extracellular matrix (ECM): ECM proteins, cell-cell receptors and cell-ECM receptors. The sum of the latter reactions lead to changes in cell behavior (such as proliferation, migration and differentiation) that are at the heart of morphogenesis (Fig. 2).

\section{Phase I: specification of the lung primordium}

The origin of the lung epithelium is the embryonic gut endoderm [1], whose emergence occurs at gastrulation
Figure 2

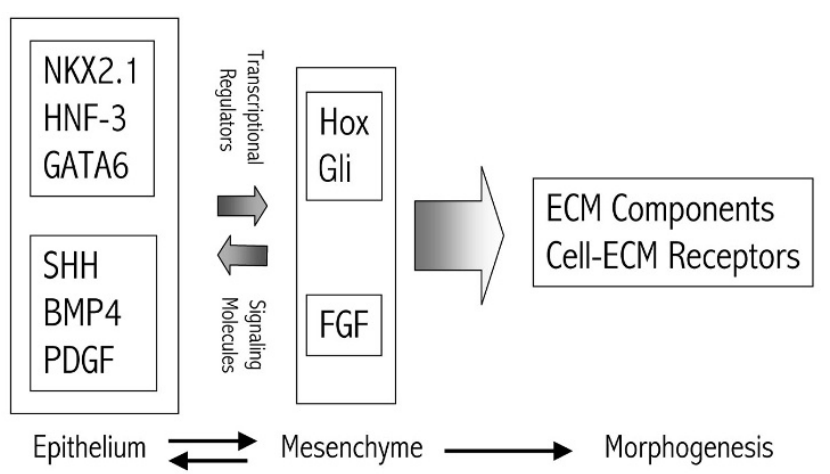

Epithelial mesenchymal interactions in lung development. Examples of the major participants are included.

from the cells within the early node and the primitive streak. The lung mesenchyme is thought to be contributed by the splanchnic mesenchyme and possibly the neural crest. The lung primordium originates from the ventral wall of the anterior foregut. Although direct information is scarce, the accumulated data indicate that a 'hierarchy' of transcriptional regulators might participate in temporal and spatial specification of the lung primordium. The first detectable transcriptional regulatory genes whose expression persists in endodermally derived adult structures is the winged-helix HNF-3 gene family whose members retain striking similarities to those of the forkhead ( $f k h$ ) gene family in Drosophila [2]. The lung primordium is specified within the boundaries of HNF-3 $\beta$ and HNF-3 $\alpha$ expression. HNF-3 $\alpha$ and HNF-3 $\beta$ are detected as early as the time at which the esophagus and laryngo-tracheal groove begin to differentiate (day 9.5 in mouse). HNF-3 $\alpha$ and HNF-3 $\beta$ are expressed in fully differentiated adult bronchial epithelium [2] and participate in the regulation of lung-specific genes such as $S p-b$ [which encodes surfactant protein (SP) B] in vitro [3]. Another member, HNF-3 $\gamma$, is detectable during hindgut differentiation and persists through liver and stomach morphogenesis.

Thus, the regionally specific expression of HNF-3 gene family might constitute a molecular axial code specifying the progenitors of the structures that emerge from the gut endoderm. Whether activation of the HNF-3 gene family is necessary or sufficient for specification of the lung primordium remains to be determined experimentally. HNF-3 $\beta^{-/-}$embryos die in early fetal development before the onset of lung morphogenesis [4]. With the exception of a single paper [5] implicating a putative $\mathrm{SHH}$-independent glial nuclear protein (Gli) pathway, little in the way of direct experimental evidence is available about the signals that specify the lung primordium. In compound homozygous Gli2;Gli3 mutants, no evidence of lung structure can be ascertained [5]. Shh encodes a morphogen, phylo- 
genetically related to hedgehog in Drosophila, which is involved in the patterning of a number of body parts in the fruitfly. SHH is expressed in the pulmonary epithelium and is thought to interact with the cellular receptor patched on mesenchymal cells. Intracellular $\mathrm{SHH}$ signaling is mediated through the activation of the Gli family of transcriptional factors, the vertebrate homologs of the Drosophila cubitus interruptus. Three Gli transcription factors are expressed in the lung mesenchyme in distinct temporal and spatial patterns. The effects of Gli2;Gli3 compound mutation seems to be independent of $\mathrm{SHH}$ signaling, because $\mathrm{Shh}^{-1-}$ mutant embryos are born with lung parenchyma, albeit abnormal [6]. It is noteworthy that specification of the lung primordium seems to be independent of almost all factors currently deemed to be crucial to subsequent normal lung morphogenesis (such as $N k \times 2.1$, Shh and Fgf-10; see below). That is, a lung rudiment, albeit abnormal, is found in functionally null mutants of all the aforementioned genes.

\section{Phase II: morphogenesis and cellular differentiation along the trachea}

Concurrently with the formation of the lung bud, the expression pattern of a homeodomain transcriptional factor NKX2.1 (otherwise known as TTF-1 or TEBP) coincides with the dorso-ventral boundary within the anterior foregut, clearly distinguishing the pulmonary primordium from that of the esophagus [7]. Early growth and development of the lung primordium results in the formation of the trachea, the most proximal pulmonary structure. Little or no information is available about the mechanisms that govern the outgrowth of the lung primordium into a tracheal tube. Even in the most severe experimentally induced phenotypes of lung abnormalities - disruptions of the FGF pathway - a tracheal tube is nevertheless formed $[8,9]$. In $N k \times 2.1^{-l-}$ lungs the trachea is markedly foreshortened and remains fused to the esophagus, resembling a relatively rare anatomical deformity in humans, known as 'complete tracheo-esophageal cleft'. Similar tracheo-esophageal phenotypes have been described for $\mathrm{Shh}^{-/}$, $\mathrm{Gli2}^{-1-} ; \mathrm{Gli3}^{+/-}$and compound retinoic acid receptor $R A R-\alpha 1^{-/-} ; R A R-\beta 2^{-/-}$mutants $[5,6,10]$. Retinoid receptors are members of the nuclear receptor superfamily that mediate the effects of signaling by retinoids. The relationship, if any, between retinoids, Shh and Nkx2.1 in the process of tracheo-esophageal septation remains to be deciphered. Shh is expressed in Nkx2.1-1- lungs; conversely, $N k \times 2.1$ can be detected in $S h h^{-/-}$lungs, suggesting independent but perhaps functionally parallel roles for the two loci in tracheal morphogenesis.

In vertebrates the trachea includes a number of phenotypically distinct cell types, including ciliated and nonciliated columnar epithelial cells and many secretory cells such as serous and goblet cells. The specific signaling and transcriptional mediators necessary for the differentiation and spatial organization of these cell types along the trachea remain largely unknown. A member of the winged-helix family of transcription factors, HNF-3/forkhead homolog-4 (HFH-4), which is expressed in the bronchial epithelium, seems to be necessary for the differentiation of ciliated epithelial cells in the lung [11]. $H f h-4$ is not specific to the lung; its expression occurs in fetal kidney, oviduct and other embryonic organs. Thus, the entire process of ciliogenesis is absent from $\mathrm{Hfh}-4^{-/-}$ embryos that also exhibit situs abnormalities [11]. Hfh-4 seems to be not only necessary but also sufficient for the differentiation of columnar ciliated epithelial cells because ectopic expression of $\mathrm{Hfh}-4$ results in the appearance of a subset of columnar cells in the distal lung, where cuboidal type II and squamous type I epithelial cells normally abound exclusively [12].

\section{Phase III: branching morphogenesis and development of the lung parenchyma}

Lung development and the differentiation of highly specialized cell types along the P-D axis require branching morphogenesis. Branching morphogenesis is dependent on epithelial-mesenchymal interactions through the activity of a complex network of molecules including peptide growth factors, transcriptional regulators and ECM proteins and their receptors [13]. In general, the nature of the interactions between the epithelial and mesenchymal compartments is based on the exchange of developmental cues that establish positional information at the level of individual cells. Depending on their developmental history, the cells base their behavior, whether it be proliferation, migration or differentiation, on the interpretation of the available positional information within a given morphogenetic boundary $\left[{ }^{\circ} 4^{\circ}\right]$. In the simplest model, developmental signals necessary for morphogenesis and cytodifferentiation can be communicated as positional information through cell-cell interactions that trigger specific patterns of gene expression by the activation of transcriptional regulators.

Currently, the crucial components of epithelialmesenchymal interactions include a number of signaling molecules and transcription factors (Fig. 2). In the lung epithelium, among a number of major signaling molecules with established roles are BMP-4, SHH and plateletderived growth factor (PDGF), although BMP-4 is also detectable in the mesenchyme late in embryonic lung development. In the lung mesenchyme, the most crucial mediator of branching morphogenesis is the FGF pathway and in particular FGF-10. In addition to the Gli family, Hox cluster transcription factors are also expressed in the lung mesenchyme. The transcription factors HNF-3, HFH-4, GATA6, N-Myc and NKX2.1 are expressed in the lung epithelium and although the evidence for HNF-3 and GATA6 is indirect, they all seem to be key regulators of epithelial-mesenchymal interactions. 


\section{Transcriptional regulators and signaling in the lung mesenchyme}

Although the role of mesenchyme in lung morphogenesis has been appreciated for a long time, the genetic dissection of mesenchyme has proved far more difficult. Classical and more recent, elegant experiments with tissue recombination have clearly shown that two functionally distinct types of mesenchyme direct tracheal (nonbranching) and parenchymal (branching) lung epithelial morphogenesis [15]. The signaling and the transcriptional factors expressed by the two mesenchyme types are presumed to be distinct, but few discrete data are currently at hand. Members of the transcriptional factors encoded by the Hox superfamily are known to be expressed in the lung mesenchyme. In particular, targeting of the Hoxa-5 gene reveals the crucial role of this family in directing epithelial morphogenesis. Hoxa-5-/mice die postnatally of abnormalities associated with morphogenesis of the trachea and decreased pulmonary surfactant production (cell differentiation) in the lung, and abnormal expression of epithelial transcription factors Nkx2.1, Hnf-3 and Myc [16]. Because transcription factors are by definition unlikely to mediate cell-cell interactions directly, potential downstream target(s) of Hoxa-5 must include signaling factors (such as FGFs) and ECM components and their receptors.

Another key mesenchymal mediator of lung development is the FGF signaling pathway. Disruption of this pathway leads to profound interruptions in lung morphogenesis. FGFs are made by the mesenchyme but act through their cognate receptors found on epithelial cells. Targeted disruption of $F g f-10$ results in a lack of lung structures distal to mainstem bronchi $[8,9]$. The role of FGF in directing epithelial morphogenesis is probably related to its ability to direct both epithelial cell proliferation and cell differentiation. Transcriptional factors activated by FGF-induced cell proliferation, such as c-fos and c-myc, have been identified and probably have similar role(s) in the proliferation of lung epithelial and mesenchymal cells. Morphoregulatory transcription factors activated in the lung by FGF-10 remain elusive. In limb morphogenesis, ectopic application of FGF leads to the activation of $S n R$, which encodes a vertebrate homolog of the Drosophila zinc-finger transcription factor Snail [17]. Other transcription factors, in particular $T b x-4$ and $T b x-5$, are also activated in response to FGF signaling in early limb specification. Members of the $T b x$ family of transcription factors are expressed in the lung epithelium [18] but their relationship to FGF signaling has not been explored. An examination of whether and how any of the latter transcriptional regulators are involved in epithelial-mesenchymal interactions during lung development is needed to elucidate the key role of the FGF signaling pathway in driving lung morphogenesis.
Transcriptional regulators and signaling in the lung epithelium; P-D morphogenesis

Throughout lung development, cell-cell interactions between the epithelial and the mesenchymal compartments of the lung are vital for normal morphogenesis. Presumably, transcriptional factors mediate the instructive and inductive signaling that arises from cell-cell interactions. For example, during lung branching morphogenesis, the reception and/or interpretation of mesenchymal signaling by epithelial cells are dependent on, among others, the normal activity of the $N$-myc locus. $N$-myc is expressed in the lung epithelium, and mouse embryos with targeted disruption of $N$-myc develop abnormal dysfunctional lungs [19]. The $N$-myc proto-oncogene encodes a basic helix-loop-helix leucine zipper transcription factor that is thought to be involved in both cellular proliferation and differentiation.

Important outcomes of cell-cell interactions are the spatial organization of the lung along the P-D axis and the differentiation of specialized epithelial cell types. The most profound disruption of lung growth and differentiation along the P-D axis occurs in Fgf-10-1- mutant embryos (see above). In mutations that allow the progression of lung beyond the mainstem bronchi, disruption of $N k \times 2.1$ renders the most severe defects in P-D morphogenesis. In $N k \times 2.1^{-1-}$ embryos, no evidence of distal lung structure beyond the secondary or tertiary bronchi exists [7]. The disruption of SHH signaling also perturbs P-D lung morphogenesis. Nevertheless, limited distal lung development and cellular differentiation are observed because cells expressing surfactant proteins can indeed be detected in $S^{-1-} h^{-l}$ lungs [6]. Thus, lung development can be viewed as a two-step process. Proximal lung morphogenesis is independent of $N k x 2.1, F g f-10$ and Shh, whereas distal lung morphogenesis is strictly dependent on $N k x 2.1$ and Fgf-10 (and somewhat dependent on Shh).

NKX2.1 is a homeodomain transcriptional regulator that is expressed in the lung, the thyroid and the ventral forebrain. In co-transfection experiments, Nkx2.1 activates the promoters of lung-specific surfactant protein genes such as $S p-b$ [3]. NKX2.1 was the first homeodomain transcriptional regulator whose suppression by antisense oligonucleotides [20] or disruption by homologous recombination in vivo [21] resulted in severely abnormal lungs, leading to postnatal death due to respiratory failure. Several lines of evidence suggest that an absence of $N k x 2.1$ activity leads to the inhibition of distal lung morphogenesis [22]. Morphologically, $N k \times 2.1^{-/-}$lung epithelium is composed solely of stratified and pseudostratified columnar epithelial cells, which are characteristically found in proximal compartments of wild type lungs. The absence of distal lung epithelial-specific differentiation markers (for example $S p-c$ and $S p-a)$, combined with evidence of ciliary differentiation and the synthesis of acid mucopolysaccharides, which are found in differentiated tracheal and bronchial epithelia, 
further suggest that $N k \times 2.1^{-1-}$ lungs are arrested in a proximal state. Importantly, the expression pattern of regionally specific, differentially spliced forms of vascular endothelial growth factor (Vegf) transcripts is uniquely proximal in Nkx2.1-/- lungs, providing further evidence that lung distal morphogenesis is inhibited in the absence of $N k x 2.1$ [22].

Given the profound abnormalities in Nkx2.1-1- lungs, it is important to know the downstream targets and upstream modulators of this critical transcriptional regulator. The expression of $B m p 4, \alpha$-integrins and collagen type IV are reduced or absent in $N k \times 2.1^{-1-}$ lungs, suggesting that they might be downstream of $N k \times 2.1$ [22]. Bmp4 is a major participant in lung distal morphogenesis whose expression is confined to the growing tip of the branching epithelium. The targeted disruption of $B m p 4$ resulted in embryonic lethality before embryonic day 10 and was therefore uninformative for lung morphogenesis [23]. The operational connection between distal lung morphogenesis and Bmp4 was also elegantly demonstrated by the ectopic expression of $X$-noggin, an inhibitor of BMP4, and a dominant-negative BMP4 receptor, Alk-6, both of which resulted in a proximalization of the lung akin to the functional deletion of $N k \times 2.1$ [24,25].

The precise role of Bmp4 in P-D lung morphogenesis and its relationship with $N k x 2.1$ requires further study. Downstream of BMP-type I receptor, the transcription factors Smad1, Smad5 and Smad8 are activated by phosphorylation, which enables them to form hetero-oligomeric complexes with Smad4 and translocate to the nucleus to mediate the transcription of Bmp4 inducible genes. Homozygous functional deletion of Smad4 results in early embryonic lethality before embryonic day 7.5 [26]. Smad5 ${ }^{-/-}$embryos die between embryonic days 9.5 and 11.5 , but lung abnormalities were not reported for these embryos [27]. Smad3 knockout animals are viable [28]. A family of homeodomain transcription factors encoded by $D / x$ genes, which represent the vertebrate homologs of Distalless in Drosophila are involved in P-D axis formation and might be activated by Bmp4 signaling [29]. At least six members of the $D / x$ family are expressed in neural tissue, bone and cartilage [30]. Interestingly, the pattern of $N k x 2.1$ and those of $D / x-1$ and $D / x-2$ overlap in the brain [31]. D/x-2 is expressed in the murine lung, but its precise function remains unknown at present $(\mathrm{C} \mathrm{Li}$ and $\mathrm{P}$ Minoo, unpublished data).

Modulators of $N k x 2.1$ have proved more difficult to identify. It is not at all clear how Nkx2.1 becomes activated in the ventral endoderm of the anterior foregut, the site of emergence of the lung diverticulum. However, this initial activation does not seem to be dependent on Shh [6] or Fgf-10 signaling (P Minoo, unpublished data). In the diencephalon, another site of $N k x 2.1$ expression, $N k x 2.1$ is activated at the three-somite stage by mechanisms that require cell-cell interactions [32]. Several studies have been directed at deciphering the regulation of $N k \times 2.1$ in cell culture experiments. These studies have been confounded by the complexity of the $N k x 2.1$ gene structure and the presence of multiple putative promoter regions. In cell culture, HNF-3 can bind to and increase the activity of the Nkx2.1 promoter [33]. However, whether HNF-3 is necessary for the activation of $N k x 2.1$ in vivo in the lung primordium remains to be elucidated.

Also of interest is the possible role of members of the zincfinger transcription factor GATA, whose consensus binding sites are present on Nkx2.1. GATA5 and GATA6 are expressed in the lung, but the expression of GATA6 coincides with $N k x 2.1$ and persists in adult alveolar type II cells [34]. Disruption of the GATA6 locus in mice leads to early embryonic lethality before the emergence of the lung primordium [35]. In addition, in GATA6 ${ }^{-/-}$;wild-type chimeras no contributions have been observed of 'green-blue' cells of GATA6-/--lacZ genotype to pulmonary epithelium, indicating that GATA6 has a crucial role in pulmonary epithelial cell lineages [35]. Because in cultured cells GATA6 participates in Nkx2.1 transcription [34], it might also be part of the mechanism in vivo that initiates the transcription of $N k x 2.1$ in the ventral endoderm. Because the $5^{\prime}$ regulatory regions of $N k \times 2.1$ contain functional NKX2.1 binding sites, it is also possible that, once activated, autoregulation might be a mechanism by which $N k \times 2.1$ expression is maintained in various cell types.

\section{Phase IV: alveologenesis and differentiation of alveolar type I and type II cells}

From a physiological standpoint, alveologenesis, the process that subdivides the lung saccular compartments into distinct alveoli, is a key step in the maturation of the pulmonary system and the establishment of effective gas exchange. FGF and PDGF signaling pathways have been implicated in alveologenesis [36,37], but transcription factors downstream of these pathways remain unknown (see also above). Thus, limited discrete information is available about the signaling mechanisms and the transcriptional factors that participate in alveologenesis. It is reasonable to speculate on a role for many of the factors involved in early lung morphogenesis whose expression persist in the mature lung. These include Hnf-3, GATA6 and $N k \times 2$.1. Direct demonstration for their involvement awaits the generation of conditional mutants.

Retinoids and glucocorticoids affect alveologenesis, but their explicit role(s) remain controversial. Retinoids are known to alter the expression pattern of the transcriptional factors belonging to the Hox gene cluster in the mesenchyme of the developing lung [38]. Such changes have the potential to alter the inductive or instructive role of the mesenchyme, thereby affecting epithelial morphogenesis. However, with the exception of Hoxa-5, neither ectopic 
expression nor loss-of-function mutations for a number of Hox genes results in grossly abnormal lung phenotypes, perhaps owing to functional redundancy. The role of glucocorticoids is also not well understood. The glucocorticoid receptor is a transcription factor belonging to the same superfamily as retinoid receptors. Functional deletion of the glucocorticoid receptor results in postnatal death due to respiratory insufficiency [39]. Detailed studies have demonstrated the presence of functional glucocorticoid binding sites on pulmonary surfactant protein genes [40]. However, embryos with mutations in the DNA binding domain of the glucocorticoid receptor that abrogate its dimerization and hence its gene activation capability have normal lungs, suggesting that the effect of glucocorticoids on lung development might not be dependent on the DNA binding and transactivation of downstream target genes [41].

The cell lineage history of alveolar type I and type II cells is unknown. Among a number of biochemical and morphological criteria, alveolar type II cells are defined by the synthesis of surfactant protein genes whose transcription is dependent on (or at least affected by) Nkx2.1. The pattern of expression of $N k \times 2.1$ during lung development suggests that $N k \times 2.1$ is selectively repressed in a P-D direction. Thus, although $N k \times 2.1$ is expressed in nearly all epithelial cells in early lung development, tracheal and bronchial epithelial cells gradually lose $N k \times 2.1$ expression as distal lung morphogenesis ensues [22]. In parallel, $N k \times 2.1$ expression is selectively maintained in a subset of cells that seem to constitute the progenitor of alveolar type II cells. The developmental mechanism(s) involved in the latter processes are unknown, but probably involve cell-cell interactions. Recent results suggest that $N k \times 2.1$ is auto-regulated ( $\mathrm{P}$ Minoo, unpublished data). A positive feedback loop as represented by autoregulation might explain the mechanism by which $N k \times 2.1$ activity is maintained in specific epithelial cell types during lung morphogenesis and in adult lung alveolar type II cells. Conversely, abrogation of this loop might explain the mechanism by which the selective repression of $N k \times 2.1$ is accomplished in a P-D direction. The transcriptional regulation of alveolar type I cell differentiation remains entirely unknown.

\section{Conclusions}

The functional linkage between morphogenetic signaling and cellular behavior is provided by the activity of transcription factors. In the lung, the two compartments of mesoderm and endodermally derived epithelium, each with distinct sets of signaling molecules and transcription factors, interact to implement the highly complex pattern of structural growth of the lungs and the differentiation of specialized epithelial cell types. In deciphering the details of lung development, valuable insights have been gained from studies of the transgenic manipulation of specific genes. These studies have also provided intriguing clues to potential overlap and interactions between various morphoregulatory molecules that participate in lung development (for examples see Shh, RAR, Gli and Nkx2.1 in tracheal development). A number of key components in lung development are also crucial to early embryogenesis and their disruption has led to embryonic lethality preceding lung development. The application of conditional mutagenesis will undoubtedly overcome this obstacle in the near future. Perhaps the most intriguing outcome of the past few years is the recognition that neither the signaling nor the transcriptional factors are uniquely lung-specific, indicating that lung specificity must arise from the unique combinatorial interactions of non-specific components. Among these, the role of the transcriptional regulator NKX2.1 is central to lung development. Although expressed in the thyroid and brain, the unique response of NKX2.1 to signaling molecules and its unique interactions with other transcription factors on morphoregulatory as well as lungspecific genes must in part underlie the specificity of normal lung morphogenesis.

\section{Acknowledgements}

I am indebted to Phil Ballard and Franco Demayo for criticisms of the manuscript and for making valuable suggestions. I ask forgiveness from colleagues whose work I might have overlooked. I am grateful for the support provided by NIH HL56590, HL60231 and the Hastings Foundation.

\section{References}

Articles of particular interest have been highlighted as:

- of special interest

- of outstanding interest

1. Ten Have-Opbroek AAW: The development of the lung in mammals: an analysis of concepts and findings. Am J Anat 1981, 162:201-219.

2. Monaghan AP, Kaestner KH, Grau E, Schutz G: Postimplantation expression patterns indicate a role for the mouse forkhead/HNF$3 \alpha, \beta$ and $\gamma$ genes in determination of the definitive endoderm, chordamesoderm and neuroectoderm. Development 1993, 119: 567-578.

3. Bohinski RJ, Di Lauro R, Whitsett JA: The lung-specific surfactant protein $B$ gene promoter is a target for thyroid transcription factor 1 and hepatocyte nuclear factor 3 , indicating common factors for organ-specific gene expression along the foregut axis. $\mathrm{Mol} \mathrm{Cell}$ Biol 1994, 9:5671-5681.

4. Ang SL, Rossant J: HNF-3 $\beta$ is essential for node and notochord formation in mouse development. Cell 1994, 78:561-574.

5. Motoyama J, Liu J, Mo R, Ding Q, Post M, Hui CC: Essential function of Gli2 and Gli3 in the formation of lung, trachea and esophagus. Nat Genet 1998, 20:54-57.

6. Pepicelli CV, Lewis ML, McMahon P: Sonic hedgehog regulates branching morphogenesis in the mammalian lung. Curr Biol 1998, 8:1083-1086.

7. Minoo P, Su G, Drum H, Bringas $P$, Kimura $S$ : Defects in tracheosophageal and lung morphogenesis in Nkx2.1(-/-) mouse embryos. Dev Biol 1999, 209:60-71.

8. Min H, Danielko DM, Scully SA, Bolon B, Rihg BD, Tarpley JE, DeRose M, Simonet WS: Fgf-10 is required for both limb and lung development and exhibits striking functional similarity to Drosophila branchless. Genes Dev 1998, 12:3156-3161. 
9. Sekine K, Ohuchi H, Fujiwara M, Yamasaki M, Yoshizawa T, Sato T, Yagishita N, Matsui D, Koga $Y$, Itoh N, Kato S: Fgf10 is essential for limb and lung formation. Nat Genet 1999, 21:138-141.

10. Mendelsohn C, Lohnes D, Decimo D, Lufkin T, LeMeur M, Chambon P, Mark M: Function of the retinoic acid receptors (RARs) during development (II). Multiple abnormalities at various stages of organogenesis in RAR double mutants. Development 1994, 120: $2749-2771$.

11. Chen J, Knowles HJ, Hebert JL, Hackett BP: Mutation of the mouse hepatocyte nuclear factor/forkhead homologue 4 gene results in absence of cilia and random left-right asymmetry. J Clin Invest 1998, 102:1077-1082.

12. Tichelaar JW, Lim L, Costa RH, Whitsett JA: HNF-3/forkhead homologue-4 influences lung morphogenesis and respiratory epithelial cell differentiation in vivo. Dev Biol 1999, 213:405-417.

13. Minoo $\mathrm{P}$, King RJ: Epithelial-mesenchymal interactions in lung development. Annu Rev Physiol 1994, 56: 13-45.

14. Wolpert L: One hundred years of positional information. Trends Genet 1996, 12:359-364.

This review and the references therein provide an extremely useful discussion on the concept of positional information.

15. Shannon JM, Nielsen LD, Gebb SA, Randell SH: Mesenchyme specifies epithelial differentiation in reciprocal recombinants of embryonic lung and trachea. Dev Dyn 1998, 212:484-494.

16. Aubin J, Lemieux M, Tremblay M, Berard J, Jeannotte L: Early postnatal lethality in Hoxa-5 mutant mice is attributable to respiratory tract defects. Dev Biol 1997, 192:432-445.

17. Issac A, Cohn MJ, Ashby P, Ataliotis P, Spicer DB, Cooke J, Tickle C: FGF and genes encoding transcription factors in early limb specification. Mech Dev 2000, 93:41-48.

18. Campbell C, Goodrich K, Casey G, Beatty B: Cloning and mapping of a human gene (TBX2) sharing a highly conserved protein motif with the Drosophila omb gene. Genomics 1995, 28:255-260.

19. Stanton BR, Perkins AS, Tessarollo L, Sassoon DA, Parada LF: Loss of $\mathrm{N}$-myc results in embryonic lethality and failure of the epithelial component of the embryo to develop. Genes Dev 1992, 6:22352247.

20. Minoo $\mathrm{P}$, Hamdan $\mathrm{H}$, Bu D, Warburton D, Stepanik $\mathrm{P}$, deLemos R: TTF-1 regulates lung epithelial morphogenesis. Dev Biol 1995, 172:694-698.

21. Kimura S, Hara Y, Pineau T, Fernandez-Salguero P, Fox HC, Ward JM, Gonzalez FJ: The T/ebp null mouse: thyroid-specific enhancerbinding protein is essential for the organogenesis of the thyroid, lung, ventral forebrain, and pituitary. Genes Dev 1996, 10:60-69.

22. Yuan B, Li C, Kimura S, Engelhardt R, Smith B, Minoo P: Inhibition of distal lung morphogenesis in Nkx2.1(-/-) embryos. Dev Dyn 2000, 217:180-190.

23. Lawson KA, Dunn NR, Roelen BA, Zeinstra LM, Davis AM, Wright CV, Korving JP, Hogan BLM: Bmp4 is required for the generation of primordial germ cells in the mouse embryo. Genes Dev 1999, 13: 424-436.

24. Bellusci S, Henderson R, Winnier G, Oikawa T, Hogan B: Evidence from normal expression and targeted misexpression that bone morphogenetic protein-4 (Bmp-4) plays a role in mouse embryonic lung morphogenesis. Development 1996, 122, 1693-1702.

25. Weaver M, Yingling JM, Dunn NR, Bellusci S, Hogan BLM: Bmp signaling regulates proximal-distal differentiation of endoderm in mouse lung development. Development 1999, 126:4005-4015.

26. Sirard C, dela Pompa JL, Elia A, Itie A, Mirtsos C, Cheung A, Hahn S, Wakeham A, Schwartz L, Kern SE, Rossant J, Mak TW: The tumor suppressor gene Smad4/Dpc4 is required for gastrulation and later for anterior development of the mouse embryo. Genes Dev 1998, 12:107-119.
27. Chang H, Huylebroeck D, Verschueren K, Guo O, Matzuk MM: Smad5 knockout mice die at mid-gestation due to multiple embryonic and extraembryonic defects. Development 1999, 126: $1631-1642$.

28. Zhu Y, Richardson JA, Parada LF, Graff JM: Smad3 mutant mice develop metastatic colorectal cancer. Cell 1998, 94:703-714.

29. Bei M, Maas R: FGFs and BMP4 induce both Msx1-independent and Msx1-dependent signaling pathways in early tooth development. Development 1998, 125:4325-4333.

30. Acampora D, Merlo GR, Paleari L, Zerega B, Postiglione MP, Mantero S, Bober E, Barbieri O, Simeone A, Levi G: Craniofacial, vestibular and bone defects in mice lacking the Distal-less-related gene DIx5. Development 1999, 126:3795-3809.

31. Bulfone A, Puelles L, Porteus MH, Frohman MA, Martin GR, Rubenstein JLR: spatially restricted expression of Dlx-1, Dlx-2 (Tes-1) Gbx-2, and Wnt-3 in the embryonic day 12.5 mouse forebrain defines potential transverse and longitudinal segmental boundaries. J Neurosci 1993, 13:3155-3172.

32. Shimamura K, Rubenstein JL: Inductive interactions direct early regionalization of the mouse forebrain. Development 1997, 124 2709-2718.

33. Ikeda K, Shaw-White JR, Wert SE, Whitsett JA: Hepatocyte nuclear factor 3 activates transcription of thyroid transcription factor 1 in respiratory epithelial cells. Mol Cell Biol 1996, 16:3626-3636.

34. Shaw-White JR, Bruno MD, Whitsett JA: GATA-6 activates transcription of thyroid transcription factor-1. J Biol Chem 1999, 274:26582664.

35. Morrisey E, Tang Z, Sigrist K, Lu M, Jiang F, Hon S, Parmacek M: GATA6 regulates HNF4 and is required for differentiation of visceral endoderm in the mouse embryo. Genes Dev 1998, 12:3579-3590.

36. Weinstein M, Xu X, Ohyama K, Deng CX: FGFR-3 and FGFR-4 function cooperatively to direct alveogenesis in the murine lung. Development 1998, 125:3615-3623.

37. Bostrom $\mathrm{H}$, Willetts $\mathrm{K}$, Penky $\mathrm{M}$, Leyeen $\mathrm{P}$, Lindhal $\mathrm{P}$, Hedstrand $\mathrm{H}$, Penka M, Hellstrom M, Gebre-Medhin S, Schalling M, Nilsson M, Kurland S, Tornell J, Heath JK, Betsholtz C: PDGF-A signaling is a critical event in lung alveolar myofibroblast development and alveogenesis. Cell 1996, 85:863-873.

38. Cardoso WV, Mitsialis SA, Brody JS, Williams MC: Retinoic acid alters the expression of pattern-related genes in the developing rat lung. Dev Dyn 1996, 207:47-59.

39. Cole TJ, Blendy JA, Monaghan P, Kriegelstein K, Schmid W, Aguzzi G Hummler E, Unsicker K, Schutz G: Targeted disruption of the glucocorticoid receptor gene blocks adrenergic chromaffin cell development and severely retards lung maturation. Genes Dev 1995, 9: 1608-1621.

40. Ballard PL, Ertsey R, Gonzales LW, Gonzales J: Transcriptional regulation of human pulmonary surfactant proteins SP-B and SP-C by glucocorticoids. Am J Respir Cell Mol Biol 1996, 14:599-607.

41. Reichardt HM, Kaestner KH, Tuchermann J, Kretz O, Wessely O, Bock R, Gass P, Schmid W, Herrlich P, Angel P, Schutz G: DNA binding of the glucocorticoid receptor is not essential for survival. Cell 1998 93:531-541.

Author's affiliation: Department of Pediatrics, Women's and Children's Hospital, University of Southern California School of Medicine, Los Angeles, California, USA

Correspondence: Parviz Minoo, Department of Pediatrics, Women's and Children's Hospital, University of Southern California School of Medicine, Los Angeles, CA 90033, USA. Tel: +1 323226 4340; fax: +1 323226 5049; e-mail: minoo@hsc.usc.edu 